\title{
Grundsätzliche Aspekte von Klima und Lufthygiene in der Region Basel
}

\section{Einleitung und Problemstellung}

Seit ca. 10 Jahren erfolgen in der Schweiz von seiten mehrerer kantonaler Luftreinhaltefachstellen wie auch von seiten des Bundes kontinuierliche Messungen der Luftschadstoffbelastung. So wird auch im Raum Basel seit 1977, ursprünglich durch das damalige Amt für Lufthygiene Baselland, an mehreren Orten die Belastung durch Schwefeldioxid gemessen (vgl. LHA, 1986). Die Meßstation des Nationalen Beobachtungsnetzes für Luftfremdstoffe auf St. Margrethen besteht seit 1980 (BUs, 1987). Mit der Inkraftsetzung der Luftreinhalte-Verordnung (LRV) im Jahre 1986 hat die Zahl der Meßstationen sowohl gesamtschweizerisch wie auch in der Region Basel noch weiter zugenommen. Alle Messungen belegen, daß in Agglomerationen und Städten die Immissionsgrenzwerte gemäß LRV zum Teil erheblich überschritten werden. Für den Sekundärschadstoff Ozon gilt diese Aussage auch für ländliche $\mathrm{Be}$ reiche weitab der Ballungszentren.

Die Luftschadstoffkonzentration in einem Gebiet oder einer Region wird durch drei Faktoren bestimmt: die Vorbelastung der Luftmassen, die in der Region selbst emittierten Schadstoffmengen und das Volumen der Luftmasse und deren Erneuerung (vgl. H. R. MOSER, 1985). Die Vorbelastung der Luftmassen, welche in eine bestimmte Region einströmen, ist abhängig von deren Nachbarschaft zu starken Emissions- oder Reinluftgebieten sowie von den Hauptwindrichtungen. Dieser Faktor ist durch die geographische Lage gegeben und kann durch lokale Maßnahmen oder politische Entscheide nicht beeinflußt werden. Das Ausmaß und die Struktur der lokalen Emissionen wird bestimmt durch die Bevölkerungszahl und -dichte, das Verkehrsaufkommen und dessen Modal-Split und die Zusammensetzung und räumliche Anordnung von Gewerbe, Industrie und öffentlichen Großemittenten wie beispielsweise einer Kehrichtverbrennungsanstalt. Dieser Faktor ist direkt beeinflußbar durch Maßnahmen und langfristige Strategien in den Bereichen technische Emissionsminderung, Verkehrs- und Siedlungsplanung.

Bei konstanter Vorbelastung und konstanter lokaler Emissionsrate bestimmt die Größe des zur Verfügung stehenden bodennahen Luftvolumens die tat- sächliche lokale Luftschadstoffkonzentration. Dieses wird definiert einerseits durch die topographischen Verhältnisse, andererseits durch die lokalmeteorologischen Bedingungen. Je enger beispielsweise ein Tal ist, um so geringer ist das zur Verfügung stehende Luftvolumen. Bei den lokalmeteorologischen Faktoren ist zu unterscheiden zwischen den horizontalen und den vertikalen Austauschbedingungen. Je stärker und ungehinderter eine Windströmung ist, um so besser erfolgt der horizontale Austausch. Dichte Überbauung erhöht die Bodenrauhigkeit und behindert so die horizontale $\mathrm{Be}$ - und Entlüftung. Der vertikale Austausch wird bestimmt durch die thermische Schichtung der Atmosphäre. Im Mittel nimmt die Temperatur in der Atmosphäre pro $100 \mathrm{~m}$ Höhenzunahme um $0,6-0,7^{\circ} \mathrm{C}$ ab. Häufig aber unterlagert eine Schicht kalter Bodenluft eine wärmere, überliegende Luftmasse. Diese bodennahe Kaltluft kann sich durch nächtliche Ausstrahlung oder dynamisch bedingt durch Absinkbewegungen in der freien Atmosphäre bilden. Die dadurch bedingte Temperaturzunahme mit der Höhe diese Inversion - bildet eine atmosphärische Sperrschicht, welche den vertikalen Austausch unterbindet und so das Luftvolumen nach oben hin begrenzt. Dieser dritte Faktor ist zwar wieder nicht direkt beeinflußbar, doch können durch raumplanerische Maßnahmen seine Auswirkungen negativ oder positiv beinflußt werden.

Im folgenden werden die topographischen und lokalmeteorologischen Voraussetzungen einerseits und die Emissionsmengen andererseits in ihrer Bedeutung für die lufthygienische Situation in der Region Basel diskutiert.

\section{Topographische Voraussetzungen und Siedlungsstruktur}

Entsprechend ihrer topographischen Lage klassiert H. WANNER (1986) die schweizerischen Städte in vier Standortstypen. Basel zählt dabei zu den Städten an topographisch geschützter Lage, welche sich an ei-

H. R. Moser, Dr. phil., Lufthygieneamt beider Basel, Rheinstr. 44, 4410 Liestal 
ner Talmündung oder an der Grenze Hügelregion/ Vorland befinden. Die Stadt Basel liegt an der Talmündung des in ost-westlicher Richtung verlaufenden Hochrheintales (4-5 km Breite) in den Oberrheingraben (ca. $200 \mathrm{~m}$ ü. M.), welcher von Basel aus in süd-nördlicher Richtung bis Frankfurt (ca. $300 \mathrm{~km}$ ) (vgl. Fig. 1) verläuft. Im Norden wird das Hochrheintal begrenzt durch den Schwarzwald und seine Vorhügel $(500-1500 \mathrm{~m}$ ü. M.), im Süden durch Tafel- und Faltenjura (500-1000 m), welcher auch die südliche Begrenzung des Oberrheingrabens bildet.

Zudem stellt das Hochrheintal und seine Seitentäler die Verbindung her zwischen dem Oberrheingraben einerseits und dem schweizerischen Mittelland andererseits. Es hat also die Funktion eines regionalmeteorologischen Ausgleichskanals zwischen zwei typischen mitteleuropäischen Beckenlandschaften, welcher vor allem bei winterlichen antizyklonalen Bedingungen als Ausfluß des Kaltluftkör-

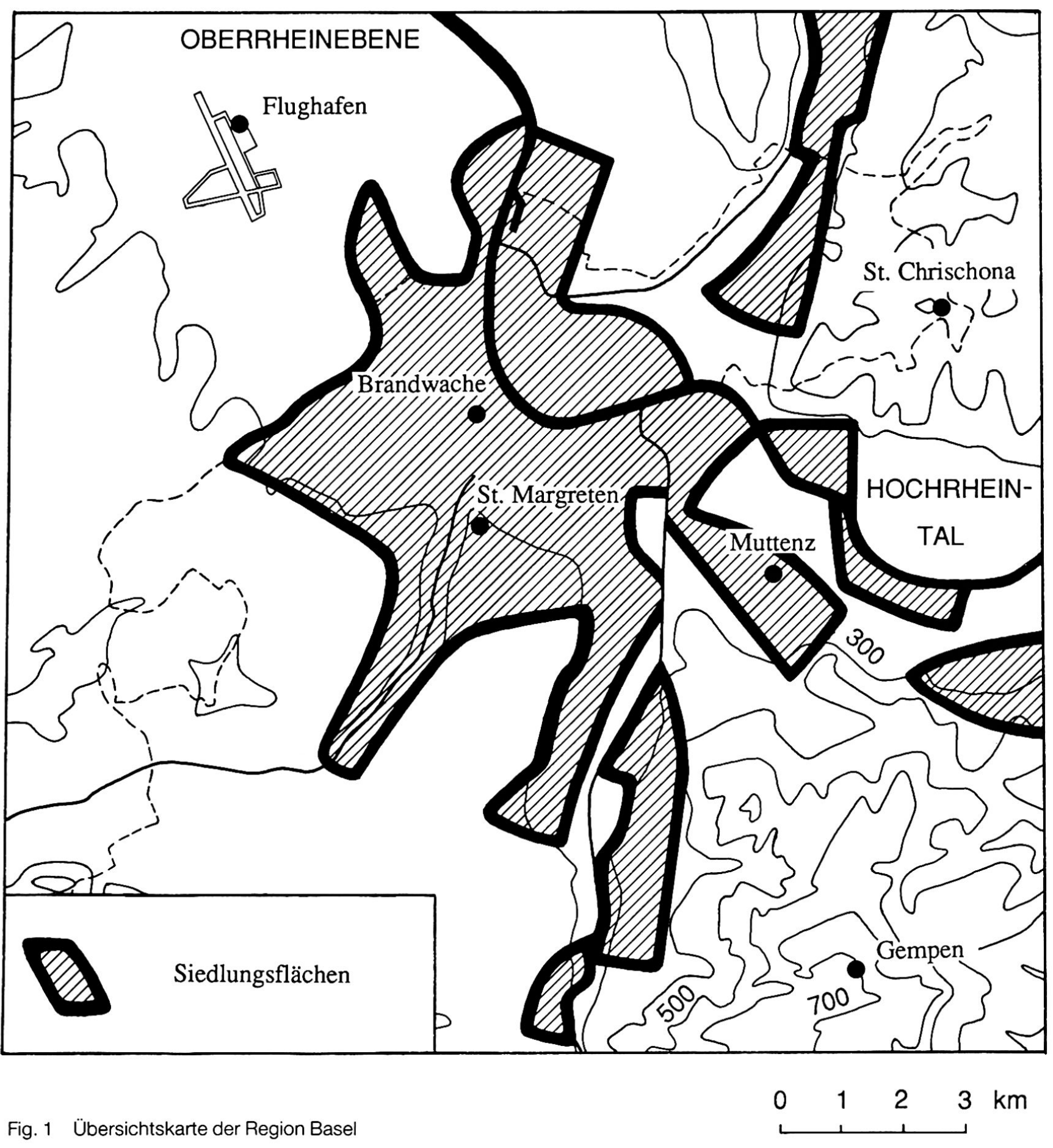


pers des schweizerischen Mittellandes in den Oberrheingraben dient (vgl. H. U. DÜTSCH, 1985).

Der Kern der Agglomeration mit der Stadt Basel als Zentrum liegt genau am Ausgang des Hochrheintales. Die Siedlungsflächen der Agglomeration schlieBen sich konzentrisch um diesen Kern an und reichen mit ihren Siedlungsbändern weit in das Hochrheintal und die nach Norden und Süden führenden Täler von Wiese, Ergolz, Birs und Birsig hinein. Dabei nehmen sie vor allem die Talböden und teilweise auch die Hanglage ein.

1984 zählte der Kanton Basel-Stadt etwas weniger als 200000 Einwohner, der Kanton Basel-Landschaft etwas mehr als 220000 Einwohner. Wie schon oben erwähnt, verteilt sich aber die Bevölkerung nicht homogen auf die gesamte Fläche der beiden Kantone, sondern konzentriert sich auf die Tallagen von Birseck und Hochrheintal sowie die Ebene um die Stadt Basel. In diesem Gebiet leben rund 350000 Einwohner, was $80 \%$ der Bevölkerung der beiden Kantone ausmacht. Dabei sind die Einwohner der angrenzenden Agglomerationsgemeinden in Frankreich und Deutschland nicht mitgezählt. Ausgedehnte Industrieflächen als Sitz von Großemittenten finden sich einerseits im Hochrheintal östlich der Stadt (Industrieareal Pratteln, Schweizerhalle, Grenzach) und im Stadtgebiet selbst (Kleinbasel, Basel Nord).

Eine weitere zusammenhängende Industriefläche erstreckt sich entlang der Birs. Auch die Verkehrsstränge folgen gebündelt den Tälern.

\section{Das Ausmaß der lokalen Emissionen}

Durch die industrielle und gewerbliche Tätigkeit, die Haushalte, die Haus- und Großfeuerungen und nicht zuletzt durch das Verkehrsaufkommen werden beträchtliche Mengen an Schadstoffen in die Luft der Region Basel abgegeben. Im folgenden werden für die Schadstoffe Schwefeldioxid, Stickoxide und die Gruppe der Kohlenwasserstoffe die emittierten Mengen in den Kantonen Basel-Stadt und BaselLandschaft kommentiert und in Fig. 2 graphisch dargestellt. Die Zahlen sind dem Zwischenbericht zum Emissionskataster Basel-Stadt (LHA, 1987) entnommen.

Die Gesamtemission an Schwefeldioxid $\left(\mathrm{SO}_{2}\right)$ ist in beiden Kantonen gleich groß und beträgt für das Stichjahr 1985 zusammen $5700 \mathrm{t} . \quad \mathrm{SO}_{2}$ entsteht hauptsächlich bei der Verbrennung von Heizöl und Kohle, bedingt durch den Schwefelgehalt dieser Brennstoffe. Die Haus- und Großfeuerungen liefern denn auch rund drei Viertel der $\mathrm{SO}_{2}$-Fracht in der Region. Die Emittenten der Quellengruppen Industrie und Gewerbe sind Abfallverbrennungsanlagen und chemische Prozesse. Der Anteil des Verkehrs ist gering und wird durch den Verbrauch von Dieseltreibstoff verursacht.
Entsprechend der Arbeitsplatzstruktur und der geringen Flächengröße ist im Kanton Basel-Stadt der prozentuale Anteil von Industrie- und Gewerbe gröBer, hingegen der Anteil des Verkehrs kleiner.

Die Stickoxid-Emissionen $\left(\mathrm{NO}_{\mathrm{x}}\right)$ in beiden Kantonen zusammen betragen beinahe $10000 \mathrm{t}$. Dabei zeigen sich zwischen beiden Kantonen sowohl Unterschiede in der Menge wie auch in der anteilmäßigen Zusammensetzung nach Emittentengruppen.

$\mathrm{NO}_{x}$ entsteht bei Verbrennungsprozessen in Motoren und Feuerungen. Im Gegensatz zum $\mathrm{SO}_{2}$ spielt hier weniger der Stickstoffgehalt der Treib- und Brennstoffe eine Rolle, sondern der immer am Verbrennungsproze $B$ beteiligte Luftstickstoff.

Der Verkehr ist der wichtigste $\mathrm{NO}_{\mathrm{x}}$-Emittent. Im Kanton Basel-Landschaft verursacht er über $80 \%$ des Ausstoßes. Im Kanton Basel-Stadt ist sein Anteil mit $50 \%$ weniger ausgeprägt, was hauptsächlich durch eine geringere Fahrleistung bedingt ist, wegen der kleinen Fläche des Kantons. Hier stechen dafür die Großfeuerungen (Kohle) und Abfallverbrennungsanlagen mehr hervor, die rund ein Drittel des $\mathrm{NO}_{\mathrm{x}}$-Ausstoßes verursachen.

Die als Kohlenwasserstoffe (HC) bezeichneten Stoffe sind organische Gase und Dämpfe und von der chemischen Zusammensetzung her sehr unterschiedlich. Zusammen mit den Stickoxiden sind sie Ausgangsprodukt für die anthropogene Bildung des Sekundärschadstoffes Ozon. Die in der Region durch menschliche Aktivität emittierten Kohlenwasserstoffmengen sind mit mehr als $20000 \mathrm{t}$ beträchtlich. Sie verteilen sich je hälftig auf die beiden Kantone, ohne daß diese sich im prozentualen $\mathrm{Mu}$ ster groß unterscheiden würden.

Die HC-Emissionen entstehen hauptsächlich bei der Verwendung von Lösungsmitteln, Farben und Lacken sowie bei chemischen Prozessen. Industrie und Gewerbe liefern auf diesem Weg knapp zwei Drittel der HC-Emission in der Region. HC-Emissionen werden auch bei Verbrennungsprozessen durch unvollständige Verbrennung verursacht. Der Verkehr liefert auf diese Art rund ein Viertel der HC-Emission in der Region.

\section{Die lokalmeteorologischen Bedingungen}

Über die lokalmeteorologischen Verhältnisse im Raum Basel liegen eine Reihe von Arbeiten vor, die sich aber zumeist mit Einzelaspekten befassen. Erwähnt seien hier nur: W. SCHÜEPP (1971, 1982), H. R. MOSER (1986) und K. KAMBER (1987). Es fehlt leider eine umfassende Arbeit, welche das Datenmaterial der ganzen Region aufbereitet, wie sie beispielsweise für Städte wie Bern, Freiburg im Breisgau oder Essen (BRD) vorliegen. In den sechziger und siebziger Jahren sind hingegen an der Abteilung für Meteorologie, Basel, eine Reihe von internen Berichten zur Frage der regionalen Durchlüftung 

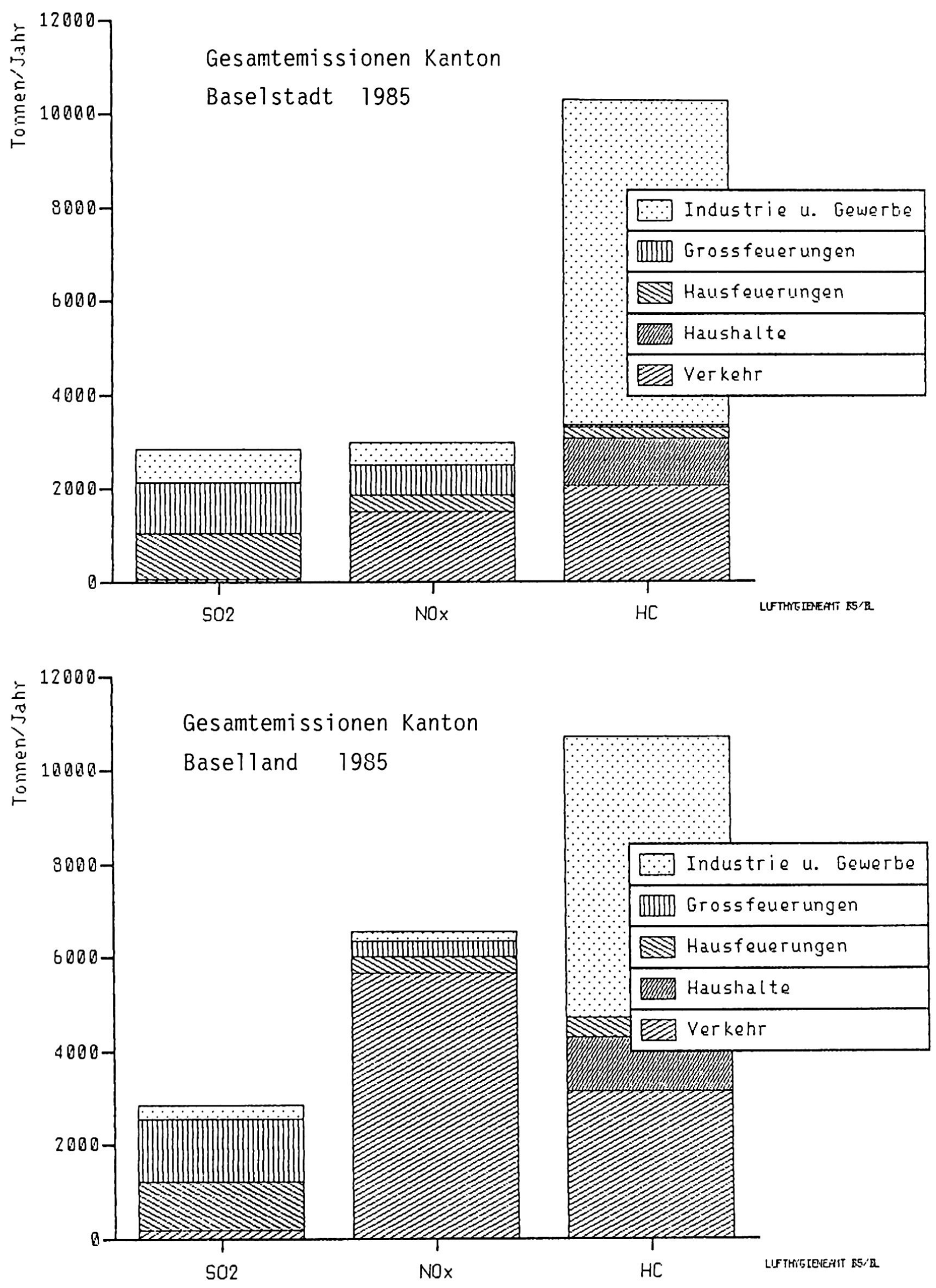

Fig. 2 Gesamtemissionen Kantone Basel-Landschaft und Basel-Stadt 1985 
und der Inversionshäufigkeit entstanden, deren Aussagen auch diesen Ausführungen zugrunde gelegt werden können (Abt. für Meteorologie, Berichte Lufthygiene, vervielfältigte Manuskripte). Leider sind aber diese Anregungen von politischer Seite damals nicht aufgenommen worden.

\section{a) Windverhältnisse}

Die Figur 3, welche die Windverhältnisse in der Region Basel zeigt, ist diesen Publikationen entnommen. Sie basiert auf Registrierungen der Jahre 1964-70. Dargestellt sind zwei Profilschnitte, der eine, horizontale, reicht vom Hochrheintal (Muttenz) über die Stadt (Brandwache) bis in die Oberrheinebene. Der andere Profilschnitt reicht vom Hochrheintal (Muttenz, $250 \mathrm{~m}$ ü. M.) über die Chrischona ( $510 \mathrm{~m}$ ü. M.) bis zum Gempen ( $710 \mathrm{~m}$ u. M. $)^{1}$ und bietet so quasi einen Vertikalschnitt durch die bodennahe Atmosphäre der Region. Dargestellt sind die Windverhältnisse, getrennt nach Winden aus dem Ostsektor $\left(50-130^{\circ}\right)$ und aus dem Westsektor $\left(230-310^{\circ}\right)$ in Form von relativen Häufigkeiten. Die absoluten Häufigkeiten der Wind- richtung und -geschwindigkeit sind zusammengestellt in Tab. 1 und 2.

Mit Ausnahme der Station Flughafen treten Winde aus den Sektoren West oder Ost mit größter Häufigkeit auf. Sie machen zusammen in der Regel um $70 \%$ aus. Dies bedeutet, daß wir es im Abschnitt Hochrheintal-Oberrheingraben mit großer Häufigkeit mit einer kanalisierten Strömung zu tun haben. Bei Westströmung wird Luft aus dem Oberrheingraben ins Hochrheintal geführt, bei Ostströmung in umgekehrter Richtung verfrachtet.

Generell läßt sich erkennen, daß beim Bodenwindfeld, wiedergegeben durch das Horizontalprofil in den Herbst- und Wintermonaten, Ostwinde überwiegen, im Frühling und Sommer hingegen eher Westwinde. Die Windgeschwindigkeiten bei Westwinden sind dabei eher größer als bei Ostwinden. Die Häufigkeit des Ostwindsektors nimmt dabei vom Hochrheintal in den Oberrheingraben ab. Dies ist Ausdruck der Tatsache, daß der Kanalisierungseffekt über der Stadt wegfällt. Einer weitergeführten Ostströmung aus dem Hochrheintal entspricht deshalb eine Winddrehung auf südöstliche bis südli-
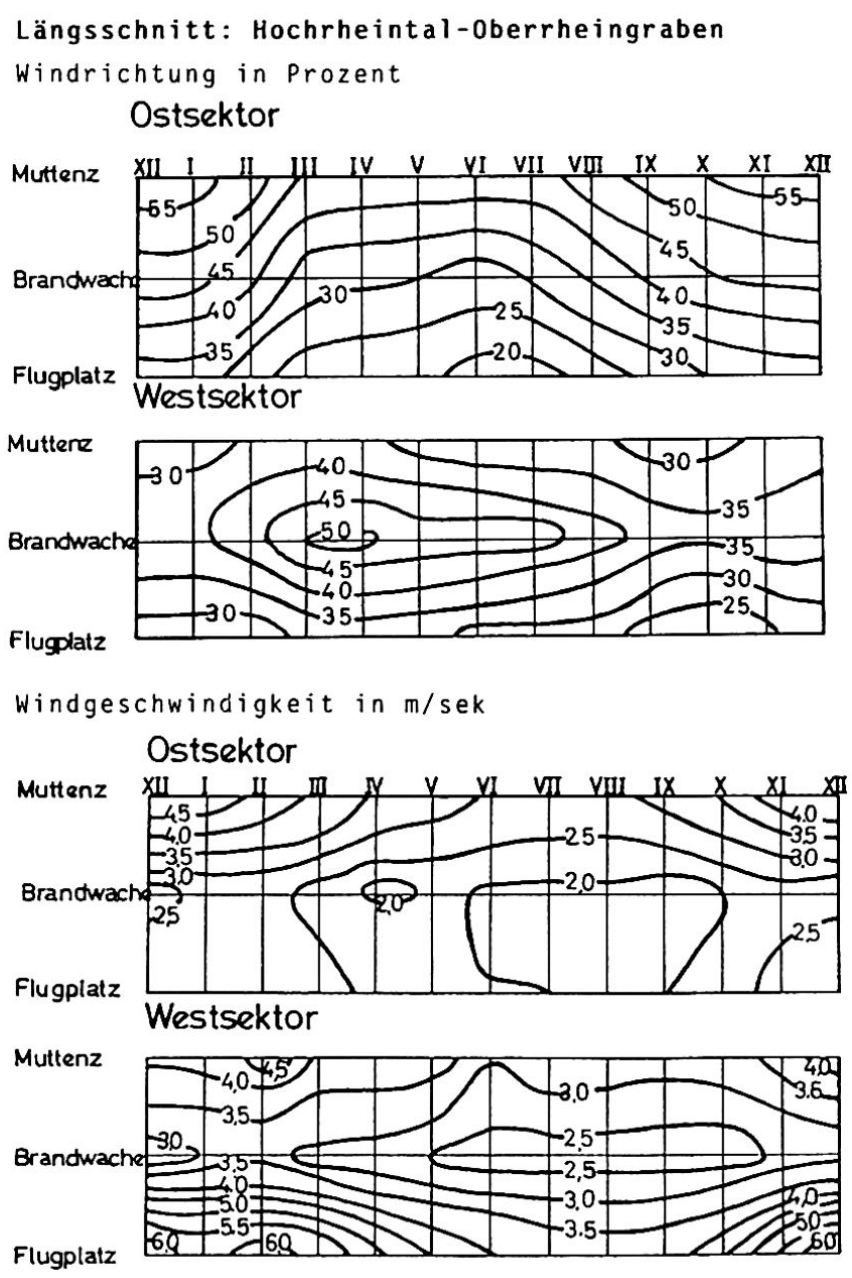

Fig. 3 Windverhältnisse im Querschnitt der Region Basel
Höhenquerschnitt: Hochrheintal-Gempen

Windrichtung in Prozent

\section{Ostsektor}

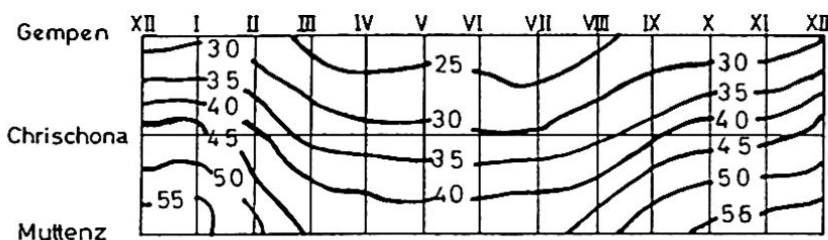

Westsektor

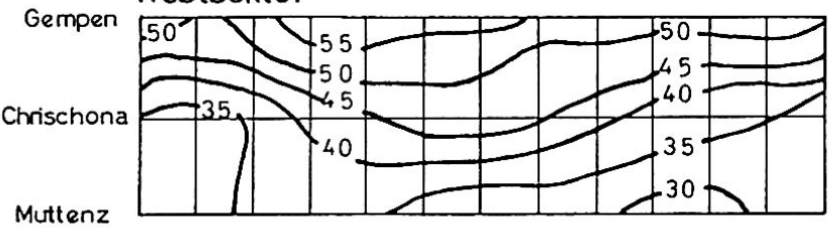

Windgeschwindigkeit in $\mathrm{m} / \mathrm{sek}$.

Ostsektor
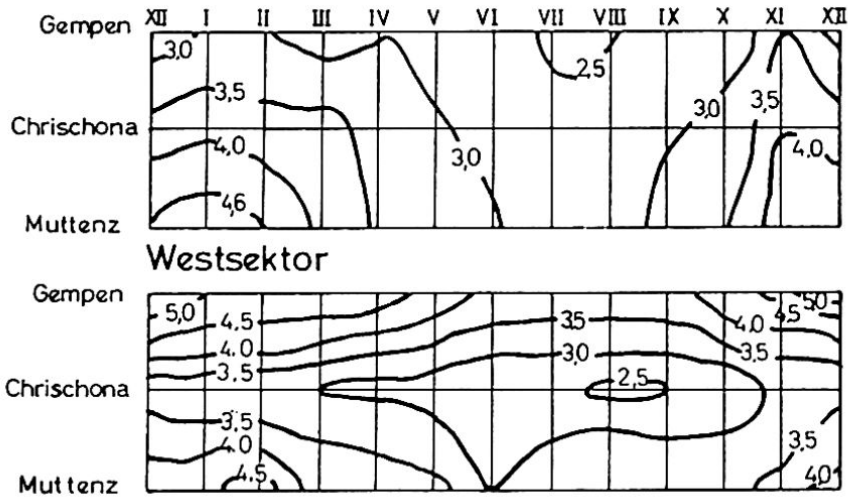
Tab. 1 Windhäufigkeiten in Promille

Windhäufigkeit o/o०

\begin{tabular}{|c|c|c|c|c|c|c|c|c|c|c|c|c|c|}
\hline Ost-Sektor & Dez. & Jan. & Febr. & März & April & Mat & Juni & Juli & Aug. & Sept. & Okt. & Nov. & Max - Miin \\
\hline Genpen & 299 & 323 & 285 & 241 & 229 & 244 & 236 & $\underline{218}$ & 246 & 266 & 256 & 264 & 95 \\
\hline Chrischona & 454 & 464 & 405 & 334 & 307 & 308 & $\underline{299}$ & 301 & 344 & 399 & 425 & 430 & 165 \\
\hline Observatorium & 415 & 444 & 388 & 317 & 294 & 279 & $\underline{256}$ & 259 & 309 & 363 & 382 & 380 & 188 \\
\hline Muttenz & 574 & 578 & 504 & 438 & 434 & 429 & 424 & 420 & 474 & 529 & 554 & 555 & 158 \\
\hline Brandweche & 466 & 478 & 409 & 327 & 304 & 301 & $\underline{286}$ & 302 & 354 & 409 & 450 & 451 & 192 \\
\hline Flugplatz & 233 & 332 & 281 & 233 & 223 & 207 & 181 & 190 & 236 & 279 & 302 & 312 & 152 \\
\hline West-Sektor & Dez. & Jan. & Febr. & Kärz & April & Hai & Juni & Juli & Aug. & Sept. & Okt. & Nov. & $\operatorname{Miax}-\operatorname{Min}$ \\
\hline Gempen & 500 & 489 & 528 & 577 & 286 & 563 & 559 & 514 & 539 & 516 & 518 & 522 & 97 \\
\hline Chrischona & 337 & 317 & 358 & 414 & 444 & 457 & 470 & 461 & 421 & 382 & 360 & 355 & 253 \\
\hline Observatoriun & 322 & 297 & 333 & 381 & 381 & 364 & 371 & 372 & 338 & 301 & 300 & 330 & 84 \\
\hline Muttenz & 333 & 329 & 364 & 384 & 363 & 339 & 324 & 316 & 304 & $\underline{286}$ & $\underline{286}$ & 321 & 98 \\
\hline Brandwache & 399 & 391 & 447 & 206 & 504 & 478 & 483 & 474 & 428 & 383 & 369 & 396 & 137 \\
\hline Flugplatz & 272 & 270 & 288 & 318 & 313 & 312 & 296 & 299 & 286 & 239 & 209 & 243 & 109 \\
\hline
\end{tabular}

Tab. 2 Windgeschwindigkeit in $\mathrm{m} / \mathrm{s}$

Windgeschwindigkeit $\mathrm{m} / \mathrm{sec}$.

\begin{tabular}{|c|c|c|c|c|c|c|c|c|c|c|c|c|c|}
\hline Ost-Sektor & Dez. & Jan. & Febr. & März & April & $\mathrm{Maj}$ & Juni & Jul & Aug. & Sept. & Okt. & Nov. & Max - Min. \\
\hline $\begin{array}{l}\text { Gempen } \\
\text { Chrischons } \\
\text { Observatorium } \\
\text { Muttenz } \\
\text { Brandwache } \\
\text { Flugplatz }\end{array}$ & $\begin{array}{l}2.9 \\
3.7 \\
2.4 \\
4.5 \\
2.4 \\
2.7\end{array}$ & $\begin{array}{l}3.1 \\
3.9 \\
\frac{2.5}{4.7} \\
\frac{2.6}{2.6}\end{array}$ & $\begin{array}{l}3.0 \\
3.6 \\
2.4 \\
4.3 \\
2.6 \\
2.6\end{array}$ & $\begin{array}{l}2.9 \\
3.6 \\
2.3 \\
3.8 \\
2.3 \\
2.7\end{array}$ & $\begin{array}{l}3.0 \\
3.2 \\
2.0 \\
3.4 \\
2.0 \\
2.3\end{array}$ & $\begin{array}{l}2.8 \\
3.0 \\
1.8 \\
3.4 \\
2.1 \\
2.3\end{array}$ & $\begin{array}{l}2.9 \\
2.7 \\
1.7 \\
2.9 \\
1.9 \\
2.0\end{array}$ & $\begin{array}{l}\frac{2.4}{2.6} \\
\frac{2.6}{2.8} \\
\frac{1.8}{2.0}\end{array}$ & $\begin{array}{l}2.5 \\
2.6 \\
\frac{1.5}{2.8} \\
\frac{1.8}{1.9}\end{array}$ & $\begin{array}{l}2.7 \\
3.0 \\
1.7 \\
3.2 \\
\frac{1.8}{2.0}\end{array}$ & $\begin{array}{l}2.8 \\
3.2 \\
1.8 \\
3.5 \\
2.0 \\
2.3\end{array}$ & $\begin{array}{l}\frac{3.5}{4.0} \\
\frac{4.0}{2.3} \\
4.4 \\
2.4 \\
2.6\end{array}$ & $\begin{array}{l}1.1 \\
1.4 \\
1.0 \\
1.9 \\
0.8 \\
0.8\end{array}$ \\
\hline West-Sektor & Dez. & Jan. & Febr. & März & April & Mat & Junt & Juli & Aug. & Sept. & Okt. & Nov. & $\operatorname{Max}-\operatorname{Min}$ \\
\hline \multirow[t]{3}{*}{$\begin{array}{l}\text { Gempen } \\
\text { Chrischona } \\
\text { Observatorium } \\
\text { Muttenz } \\
\text { Brandwache } \\
\text { Flugplatz }\end{array}$} & $\begin{array}{l}\frac{5.5}{3.3} \\
3.1 \\
4.2 \\
2.6 \\
6.3\end{array}$ & $\begin{array}{l}5.0 \\
3.3 \\
3.0 \\
4.2 \\
3.1 \\
5.7\end{array}$ & $\begin{array}{l}5.2 \\
3.1 \\
\frac{3.2}{4.6} \\
\frac{3.2}{6.2}\end{array}$ & $\begin{array}{l}5.1 \\
3.0 \\
3.1 \\
3.8 \\
2.9 \\
5.7\end{array}$ & $\begin{array}{l}4.7 \\
2.8 \\
2.9 \\
3.9 \\
2.8 \\
5.0\end{array}$ & $\begin{array}{l}4.4 \\
2.8 \\
2.6 \\
3.6 \\
2.5 \\
4.4\end{array}$ & $\begin{array}{l}3.7 \\
2.6 \\
2.3 \\
3.0 \\
2.3 \\
4.0\end{array}$ & $\begin{array}{l}3.7 \\
2.6 \\
2.4 \\
3.5 \\
2.3 \\
3.8\end{array}$ & $\begin{array}{l}\frac{3.6}{2.4} \\
\frac{2.3}{3.4} \\
2.3 \\
3.8\end{array}$ & $\begin{array}{l}3.7 \\
2.5 \\
2.2 \\
3.3 \\
\frac{2.1}{3.7}\end{array}$ & $\begin{array}{l}4.3 \\
2.6 \\
2.3 \\
3.4 \\
2.2 \\
4.5\end{array}$ & $\begin{array}{l}5.1 \\
3.2 \\
2.9 \\
3.6 \\
2.6 \\
5.5\end{array}$ & $\begin{array}{l}1.9 \\
0.9 \\
1.0 \\
1.6 \\
1.1 \\
2.6\end{array}$ \\
\hline & \multirow[b]{2}{*}{ Dez. } & \multirow[b]{2}{*}{ Jan. } & \multirow[b]{2}{*}{ Febr. } & \multicolumn{6}{|c|}{ Häufigkeit der Kalmen $\quad$ /oo } & \multirow[b]{2}{*}{ Sept. } & \multirow[b]{2}{*}{ Okt. } & \multirow[b]{2}{*}{ Nov. } & \\
\hline & & & & März & April & Mat & Junt & Jul1 & hug. & & & & \\
\hline Gempen & 36 & 39 & 21 & 26 & 20 & 17 & 26 & 27 & 33 & 30 & 48 & 25 & \\
\hline Chrischona & 32 & 73 & 68 & 43 & 28 & 36 & 29 & 37 & 48 & 34 & 51 & 46 & \\
\hline Observatorium & 57 & 91 & 66 & 57 & 36 & 55 & 52 & 62 & 85 & 97 & 97 & $n$ & \\
\hline Muttenz & 0 & 7 & 7 & 7 & 13 & 5 & 8 & 11 & 9 & 11 & 11 & 6 & \\
\hline Brandwache & 43 & 36 & 21 & 13 & 23 & 34 & 48 & 52 & 50 & 63 & 41 & 42 & \\
\hline Flugplatz & 268 & 294 & 169 & 279 & 163 & 218 & 262 & 237 & 244 & 252 & 262 & 186 & \\
\hline
\end{tabular}


che Strömung im Bereich des Flughafens, also wieder entsprechend der orographischen Lage des Oberrheingrabens. Deutlich ist vor allem auch bei Ostwind eine Abnahme der Windgeschwindigkeit über der Stadt und der Oberrheinebene. H. WANNER/ J.-A. HERTIG (1984) haben zeigen können, daß dies mit dem thermischen und dynamischen Einfluß der städtischen Überbauung zusammenhängt.

Neben dem jahreszeitlichen Gang in der Hauptwindachse der Region Basel besteht aber auch ein tageszeitlicher Gang. So treten beispielsweise an der Station Muttenz im Sommer Ostwinde mit bis 50prozentiger Häufigkeit zwischen $10 \mathrm{Uhr}$ nachts und $10 \mathrm{Uhr}$ morgens auf. Über Mittag sinkt deren Häufigkeit auf weniger als 20 Prozent, bei einem Anteil von bis zu 30 Prozent Häufigkeit an Westwinden. Im Winter bleibt hingegen die Ostwindströmung auch tagsüber meistens erhalten. Lufthygienisch hat dieses tagesperiodische Windfeld zur Folge, daß im Sommer nachts belastete Luft nach Westen und in den Oberrheingraben geführt wird, welche tagsüber unter Umständen wieder ins Hochrheintal zurückgepumpt wird. Ist dies der Fall, so findet keine durchgreifende Lufterneuerung statt.

Der vertikale Profilschnitt zeigt eine Abnahme der Bedeutung der Ostwindströmung mit zunehmender Höhe. Bei Muttenz beträgt sie um 50 Prozent, auf dem Gempen noch weniger als 30 Prozent. Wiederum sind westliche Richtungen im Sommer in allen Höhenlagen häufiger als im Winter. Generell ist bei östlichen Windrichtungen die Geschwindigkeit kleiner, wobei sie noch nach oben hin abnimmt. Bei westlicher Windrichtung ist die Geschwindigkeit größer und nimmt mit der Höhe tendenziell eher $\mathrm{zu}$.

Aus dieser unterschiedlichen Häufigkeit der Windrichtungen mit der Höhe läßt sich der Schluß ziehen, $\mathrm{da} B$ in den verschiedenen Höhenniveaus, deren Trennlinie oft um $500 \mathrm{~m}$ liegt, teilweise sich schneidende oder sogar entgegengesetzte Windrichtungen auftreten können. Am häufigsten ist diese Erscheinung im Winter und während der Nachtstunden.

\section{b) Inversionsvorkommen}

Währenddem die Bodenwindverhältnisse im Prinzip das Austauschvolumen in horizontaler Richtung definieren, legt die Höhenlage von Inversionen und deren Häufigkeit und Ausdauer das Austauschvolumen in vertikaler Richtung fest. Auch zu dieser Fragestellung gibt es in den oben erwähnten Berichten der Abteilung für Meteorologie einige Untersuchungen.

In Tab. 3 ist die Häufigkeit des Auftretens von Inversionen am Nachmittag (15.30 Uhr) im Winterhalbjahr zusammengestellt. Die Angaben basieren auf Messungen der Jahre 1964-65 und sind umgerech-
Tab. 3 Jahreszeitliche Verteilung der Häufigkeit langdauernder Inversionen

Anzahl Inversionstage (1964/65, berechnet auf 1 Jahr). Angabe des Stationspaares mit der untersten Inversion.

\begin{tabular}{lllll} 
& B-Ch & Ch-G & G-F & Total \\
Oktober & 2 & 5 & 2 & 9 \\
November & - & 2 & 1 & 3 \\
Dezember & 1 & 6 & 1 & 8 \\
Januar & 2 & 7 & 5 & 14 \\
Februar & 1 & 1 & 1 & 3 \\
Mär & 1 & - & - & 1 \\
\cline { 3 - 5 } Total Tage & 7 & $\geq 21$ & $\geq 10$ & $\geq 38$
\end{tabular}

$$
\begin{aligned}
& B=\text { Basel-Binningen }(317 \mathrm{~m}) \\
& C h=\text { Chrischona }(510 \mathrm{~m}) \\
& G=\text { Gempen }(710 \mathrm{~m}) \\
& F=\text { Feldberg }(1450 \mathrm{~m})
\end{aligned}
$$

net auf die Häufigkeit pro Jahr. Damit werden alle Inversionen erfaßt, welche auch tagsüber andauern und damit lufthygienisch durch ihren stetigen Akkumulationseffekt von großer Bedeutung sind. Die Zahl nächtlicher Inversionen, welche sich tagsüber auflösen, wird noch um ein Vielfaches größer sein. In Fig. 4 ist zudem die tageszeitliche Häufigkeit von Inversionen zwischen dem Observatorium BaselBinningen und dem Gempen für das Jahr 1964 dargestellt.

Danach treten tagsüber andauernde Inversionen hauptsächlich im Winterhalbjahr und vereinzelt im Herbst auf. Im Sommer lösen sie sich, durch die stärkere Einstrahlung bedingt, zwischen 10 und $18 \mathrm{Uhr}$, also nachmittags immer wieder auf. Ihre größte Häufigkeit mit 21 Tagen pro Jahr haben sie in der Höhenlage zwischen 500 und $700 \mathrm{~m}$. Sie treten am häufigsten auf bei Hochdrucklage sowie bei Föhnsituationen und bei Höhenwinden aus südlichen Richtungen.

Zusammenfassend läßt sich also feststellen, daß die Region Basel lokalmeteorologisch gekennzeichnet ist durch:

- ein kanalisiertes Bodenwindfeld mit tages- und jahreszeitlichen Windwechseln. Am häufigsten treten dabei kanalisierte Ostwinde auf. Halten sie auch tagsüber an, so haben sie zur Folge, daß sich die Luftschadstoffe auf ihrem Weg bis an die nördliche Grenze der Agglomeration akkumulieren und die «Urban Plume» der Stadt Basel als Ganzes in den Sundgau verlagert wird.

- eine häufige Abkoppelung vom topographisch beeinflußten Bodenwindfeld und synoptisch bedingter Windströmung in der Höhe

- die Häufigkeit von Inversionen mit all ihren negativen Folgen für die Austauschbedingungen. Ihre größte Häufigkeit liegt höher als $500 \mathrm{~m}$ ü. M., womit auch die Emissionen der Hochkamine unterhalb dieser Sperrschicht bleiben. 
Diese generellen Anregungen lassen sich auch wiederfinden bei der Analyse von Einzelfällen. So war beispielsweise die Periode mit erhöhter $\mathrm{SO}_{2}$-Belastung im Januar 1985 vor allem in ihrer ersten Phase gekoppelt mit einer schwachen, aber beständigen Ostwindströmung. Konsequenterweise traten die Höchstwerte an der nördlich der Stadt Basel gelegenen Station Flughafen auf. In der zweiten Phase hingegen nahm die Windgeschwindigkeit noch weiter $a b$, und bei stagnierenden oder wechselnden Winden traten die Maximalwerte an der Station Birsfelden auf, welche zwischen der Stadt Basel und den Agglomerationsgemeinden im Hochrheintal liegt. Im Januar 1987 hingegen herrschte eine schwache Strömung aus Richtung Norden vor, d. h. vom Oberrheingraben her.

\section{Folgerungen}

H. WANNER (1986) nennt als mögliche Maßnahme zur Verbesserung des lufthygienischen Wirkungskomplexes in den Städten zwei Bereiche:
1. Der Luftaustausch innerhalb des Stadtgebietes und zwischen der Stadt und ihrem ländlichen Umland muß gewährleistet werden.

2. Der Anteil an festen, flüssigen und gasförmigen Luftfremdstoffen muß reduziert werden.

Die erste Forderung hat die Sicherstellung einer ausreichenden Lufterneuerung zum Ziel. Dazu zählen:

- Das Freihalten von Schneisen im Siedlungskörper, welche zur Frischluftzufuhr dienen können. Damit kann die Lufterneuerung im Stadtkörper unterstützt werden.

- Die optimale Orientierung der Baukörper nach den vorherrschenden Winden im Bodenströmungsfeld und die Begrenzung der Baukörperhöhe auf wenige Stockwerke. Beide Maßnahmen verhindern eine noch stärkere Abschwächung der Windströmung über dem Siedlungsgebiet.

- Topographisch vorgegebene Strömungssysteme dürfen nicht durch ungünstige Baukuben be- oder sogar verhindert werden. Sie sorgen für eine Lufterneuerung aus dem Umland in die Stadt und treten vor allem nachts auf. Gerade diese nur schwa-

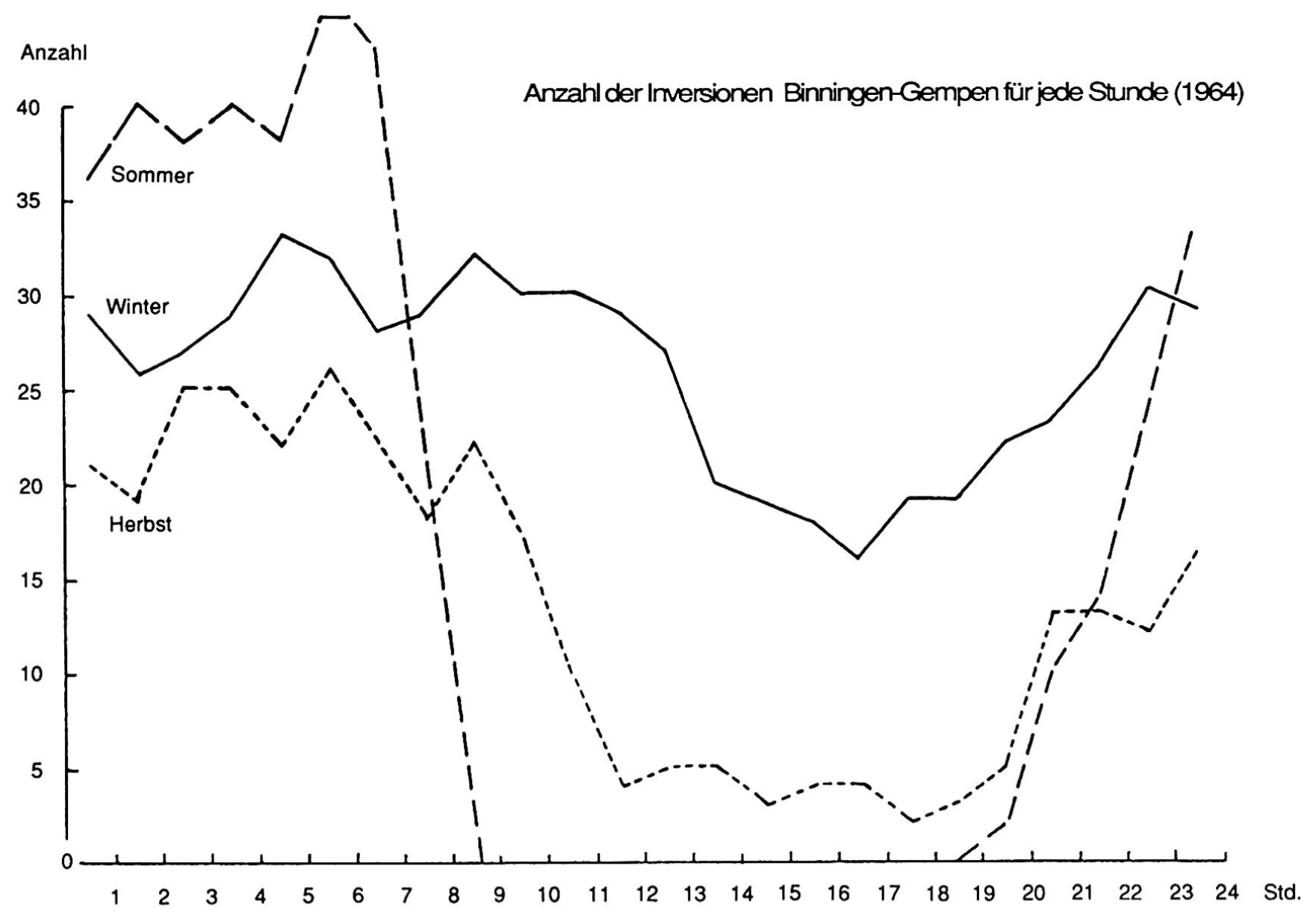

Fig. 4 Tagesperiodischer Gang der Inversionshäufigkeit 1964 
chen Windströmungen sind allerdings wegen ihrer nur geringen Mächtigkeit anfällig auf Störungen.

Diesen Forderungen kommt vor allem eine präventive Funktion zu. Sie sorgen dafür, daß das natürlich vorgegebene topographisch bedingte Austauschvermögen eines Gebietes nicht noch weiter unnötig reduziert wird. Allerdings darf die Wirksamkeit solcher Maßnahmen nicht überschätzt werden.

Umgesetzt auf den Raum Basel, müßten daraus die folgenden Forderungen abgeleitet werden:

Das grundsätzliche Ziel muß sein, zu verhindern, daß die durchgängige Strömung zwischen Hochrheintal und Oberrheingraben noch weiter erschwert wird oder sogar abbricht. Dazu dürfen:

- das Hochrheintal nicht durch querstehende, großkubige Bauten verstellt werden

- die Ausgänge der Seitentäler ins Hochrheintal nicht flächenhaft überbaut werden

- windparallele Schneisen durch die Stadt nicht durch Querriegel überbaut werden. Beispiele dazu sind der Rhein, die Gleiskörper von SBB und DB, die Freifläche zwischen Riehen und Basel

- radiale Windachsen aus den Seitentälern in Richtung Stadtkern nicht überbaut oder müßten durch zusammenhängende Grüngürtel erst geschaffen werden.

Solche raum- und siedlungsplanerischen Grundsätze können nur langfristig wirksam werden. Zudem können sie auch bei optimaler Ausnutzung nicht das Problem der übermäßigen Emissionen lösen. Trotzdem sind sie als langfristige Strategie zu verfolgen, zumal diese Maßnahmen nicht nur den lufthygienischen Wirkungskomplex günstig beeinflussen, sondern in allererster Linie auch den thermischen Wirkungskomplex, d. h. die Überwärmung der Stadt, mindern.

Gerade zu den Fragen der lufthygienisch wirksamen lokalmeteorologischen Austauschbedingungen herrscht allerdings in der Region Basel noch ein groBes Forschungsdefizit. Zwar sind die grundsätzlichen Mechanismen entlang der regionalen Hauptwindachse Hochrheintal-Oberrheingraben bekannt. Es fehlen aber Angaben über die Windverhältnisse aus den Seitentälern, dreidimensionale Untersuchungen zu möglicherweise vorhandenen stadtbürtigen Flurwindsystemen, und auch das Windregime im Birseck ist nur wenig bekannt. Solche Untersuchungen haben mit zwei Stoßrichtungen zu erfolgen:

1. Darstellung der mittleren Bodenwindverhältnisse in einer Durchlüftungskarte und der typi- schen Einzelfälle in einem Katalog der regionalen Bodenwindfelder.

2. Die regionalen Austauschverhältnisse sind als $L o$ kalwetterlagenkatalog zu definieren und diese wiederum mit den synoptisch bedingten Großwetterlagen zu verknüpfen. Erst damit werden lufthygienisch kritische Austauschbedingungen prognostizierbar. Ihre Prognostizierbarkeit ist aber Voraussetzung für die Handhabung von Smogalarmplänen!

1 Die Meßstandorte sind in Fig. 1 eingetragen

\section{Literatur}

BIDER, M. (1966): Bericht über die Ergebnisse meteorologischer Untersuchungen 1964-1965 bei Basel, im Hinblick auf die Luftverunreinigung. Abt. für Meteorologie, Basel.

Bundesamt für Umweltschutz (1987): Luftbelastung 1986 Meßresultate des Nationalen Beobachtungsnetzes für Luftfremdstoffe (NABEL). Schriftenreihe Umweltschutz Nr.67, Bern.

DÜTSCH, H. U. (1985): Large-Scale domination of a regional circulation during winter-time anticyclonic conditions. In: Met. Rundschau 38, S. 65-75.

KAMBER K. (1987): Lokalklimatische Untersuchungen im Hochrheintal vor Basel im Hinblick auf die Luftverschmutzung und damit zusammenhängende Raumordnungsprobleme. In: Regio Bas. XXVIII/1 + 2, S. 123-131.

Lufthygieneamt beider Basel (1986): Schwefeldioxid-Immissionsmeßnetz beider Basel - Meßresultate des Jahres 1985 Liestal.

Lufthygieneamt beider Basel (1987): Emissionskataster Basel-Stadt/Basel-Landschaft - Zwischenbericht, Liestal.

MOSER, H. R. (1985): Beziehungen zwischen Klima und Lufthygiene. In: Regio Bas. XXVI/3, S. 213-222.

MOSER, H. R. (1986): Stadtklimatische Aspekte der Agglomeration Basel. In: Regio Bas. XXVII/3, S. 199-209.

SCHÜEPP, W. (1971): Regionalplanung als meteorologisches Problem. In: Regio Bas. XII/1, S. 189-201.

SCHÜEPP, W. (1982): Untersuchungen über die Windverhältnisse in der Nordwestschweiz. In: Geogr. Helv. 37/4, S. $208-214$.

WANNER, H./HERTIG, J.-A. (1984): Studies of Urban Climates and Air Pollution in Switzerland. In: J. of Climate and Appl. Met., 23, S. 1614-1625.

WANNER, H. (1986): Die Grundstrukturen der städtischen Klimamodifikation und deren Bedeutung für die Raumplanung. Jahrbuch d. Geogr. Ges. von Bern, Band 55/ 1983-1985, Bern, S. 67-84. 